\title{
ÉLÉMENTS ALGÉBRIQUES SUR LE CORPS DES SÉRIES FORMELLES GÉNÉRALISÉES EN CARACTÉRISTIQUE FINIE
}

\author{
ALI BENHISSI
}

\section{Introduction}

Soient $K$ un corps commutatif et $G$ un groupe abélien totalement ordonné. Nous donnons des moyens de construction d'éléments algébriques sur le corps $K((G))$ des séries formelles généralisées à supports bien ordonnés.

Nous nous sommes inspirés des travaux de Stephanescu [7] et [8] sur les séries formelles usuelles dans plusieurs endroits de cet article.

Dans tout l'article, $G^{-}=\{\alpha \in G ; \alpha<0\}$ et $\bar{K}$ désigne une clôture algébrique de $K$. Si $f$ est une série formelle généralisée, on note $v(f)$ sa valuation naturelle et $S(f)$ son support.

\section{§1. Généralisation de la méthode de Rayner-Ribenboim}

Nous reprenons les définitions et notations de Rayner dans [4] et [5]. Soient $\mathscr{L}$ l'ensemble des sous corps de $\bar{K}$ de degré fini sur $K, \Delta$ l'enveloppe divisible de $G$ et $\mathscr{P}(G)$ la plus petite famille-corps de $\Delta$ contenant l'ensemble $\mathscr{B}(G)$, des parties bien ordonnées de $G$.

Plus précisément, désignons par $\mathscr{A}$ l'ensemble des parties $A$ de $\Delta$ telles que le sous groupe $\langle G \cup A\rangle$ de $\Delta$ engendré par $G \cup A$ est de type fini modulo $G$; c'est à dire $\langle G \cup A\rangle=G+\sum_{i=1}^{r} \mathrm{Z}_{i}$, avec $\alpha_{i} \in A$ et $\mathscr{P}_{1}(G)=\left\{\frac{S}{n} ; S \in \mathscr{B}(G)\right.$, $\left.n \in \mathrm{N}^{*}\right\}$. On a $\mathscr{P}(G)=\mathscr{P}_{1}(G) \cap \mathscr{A}$.

L'ensemble $K(\mathscr{P}(G))=\{f \in K((\Delta)) ; S(f) \in \mathscr{P}(G)\}$ est un sous corps de $K((\Delta))$ contenant $K((G))$.

1.1. Proposition. Le corps $C=\bigcup_{L \in \mathscr{L}} L(\mathscr{P}(G))$ est une clôture algébrique de $K((G))$ dans chacun des deux cas suivants: 
a) la caractéristique de $K$ est nulle.

b) K est parfait de caractéristique $p \neq 0$ et $G$ est $p$-divisible.

Démonstration. $C$ est hensélien pour sa valuation naturelle $v$, d'après [4], lemme 2 et algébrique sur $K((G))$ d'après [5] th.3.

Le cas a) résulte de [6], lemme 5.1.(i).

Supposons dans b) que $\mathrm{C}$ ne soit pas algébriquement clos. D'après [6], lemme 5.1.(ii), il existe $f \in C, v(f)<0$, tel que le polynôme $X^{p}-X-f$ soit irréductible sur $C$. On a: $f=c+f_{1}+f_{2}$, avec $c \in \bar{K}, v\left(f_{1}\right)>0$ et $S\left(f_{2}\right) \subset G^{-}$. Les polynômes : $X^{p}-X-f_{i}, i=1,2$ admettent les séries $g_{1}=-\sum_{i=0}^{\infty} f_{1}^{p^{i}}$ et $g_{2}=\sum_{i=1}^{\infty} f_{2}^{p^{-i}}$ pour racines dans $C$. Notons que $S\left(g_{2}\right) \subseteq \bigcup_{i=1}^{\infty} p^{-i} S\left(f_{2}\right)$, qui est bien ordonné, d'après [6], p.133. On trouve une contradiction.

L'exemple suivant montre qu'en caractéristique non nulle, si les conditions de (b) ne sont pas vérifiées, le corps $C$ n'est plus algébriquement clos.

Exemple. On suppose que caract $K=p \neq 0$. Soient $a \in K, \alpha \in G^{-}$et $f=\sum_{i=1}^{\infty} a^{p^{-i}} T^{p^{-i} \alpha}$. On a $f^{p}-f-a T^{\alpha}=0$.

Si $\sqrt[p]{a} \notin K$ ou $\alpha$ n'est pas $p$-divisible, alors $f \notin C$.

\section{§2. Critères d'algébricité en caractéristique non nulle}

On suppose dans le reste de l'article que la caractéristique de $K$ est $p \neq 0$. Soient $K^{p^{-\infty}}=\bigcup_{n=0}^{\infty} K^{p^{-n}}$ la clôture radicielle de $K, \mathscr{L}$ l'ensemble des sous corps de $\bar{K}$ de degrés finis sur $K^{p^{-\infty}}$ et $G^{\prime}=\left\{p^{-n} \alpha ; n \in \mathrm{N}, \alpha \in G\right\}$. Alors $\tilde{K}=\bigcup_{L \in \mathscr{L}} L\left(\mathscr{P}\left(G^{\prime}\right)\right)$ contient une clôture algébrique de $K((G))$.

2.1. Exemples de séries de Neumann algébriques. Soit $f \in \tilde{K}$ une série algébrique sur $K((G))$ de valuation $v(f)>0$. Il résulte de [1], th. p.268, que si $\sum_{n=0}^{\infty} a_{n} X^{n}$ est une série de $\bar{K}[[X]]$ algébrique sur $\bar{K}[X]$, alors la série de Neumann $\sum_{n=0}^{\infty} a_{n} f^{n}$ est algébrique sur $K((G))$.

a) Si $q$ est une puissance de $p$ et $g=\sum_{n=0}^{\infty} f^{q^{n}}$, alors $g^{q}-g+f=0$. 
b) Si $a_{n}$ est la somme, réduite modulo $p$, des chiffres de $n$, en base $p$, et $g=\sum_{n=0}^{\infty} a_{n} f^{n}$, alors $\left(f^{p}-1\right)(f-1) g^{p}-(f-1)^{2} g+f=0$.

c) Soient $s \in \mathrm{N}^{*}$ et $g=\sum_{n=0}^{\infty}\left(C_{2 n}^{n}\right)^{s} f^{n}$. On a: $g^{p-1}=\left[\sum_{i=0}^{\frac{p-1}{2}}\left(C_{2 i}^{i}\right)^{s} f^{i}\right]^{-1}$.

On suppose maintenant que $p=2$. Soit $g=\sum_{n=0}^{\infty} a_{n} f^{n}$, où $\left(a_{n}\right)$ est une suite d'éléments de $F_{2}$, définie dans [2] pour chacun des exemples suivants:

d) $\mathrm{Si}\left(a_{n}\right)$ est la suite de Rudin-Shapiro, alors: $(1+f)^{5} g^{2}+(1+f)^{4} g+$ $f^{3}=0$.

e) $\mathrm{Si}\left(a_{n}\right)$ est la suite de Baum et Sweet, alors: $g^{3}+f g+1=0$.

f) $\mathrm{Si}\left(a_{n}\right)$ est la suite de pliage de papier, alors: $f(1+f)^{4} g^{2}+(1+f)^{4} g+$ $1=0$.

Notation. Dans tout le reste de l'article $q$ désigne une puissance de $p$ et $d$ un entier naturel non divisible par $p$.

2.2. Lemme. Soit $f=\sum_{s \in S} a_{s} T^{\frac{\alpha s}{d q^{s}}}$ une série de $\tilde{K}$, où $n_{s} \in \mathbf{Z}$ et $\alpha_{s} \in G^{-}$.

On suppose que $\alpha_{s} n$ 'est pas $q$-divisible pour tout $s \in S$ et $\alpha_{s}-\alpha_{t} n$ 'est pas $q$ divisible dès que $\alpha_{s} \neq \alpha_{t}$.

Si f est algébrique sur $K((G))$, alors il existe $n \in \mathrm{N}$ et $h \in \bar{K}\left(\left(\frac{1}{d} G\right)\right)$ tels que: $h, f, f^{q}, \ldots, f^{q^{n}}$ soient linéairement dépendants sur $K$.

DÉmonstration. Il existe $n \in \mathrm{N}$ et $g, g_{0}, \ldots, g_{n} \in K((G))$ non tous nuls tels que: $g_{0} f+g_{1} f^{q}+\ldots+g_{n} f^{q^{n}}=g$. On peut supposer que: $g_{i}=b_{i}+T^{\alpha} h_{i}$, avec les $b_{i} \in K$ non tous nuls, $\alpha>0$ et $v\left(h_{i}\right) \geq 0$.

On a: $\sum_{i=0}^{n} b_{i}\left(\sum_{s \in S} a_{s}^{q^{i}} T^{\frac{\alpha s}{d q^{q_{s}-i}}}\right)+T^{\alpha} \sum_{i=0}^{n} h_{i}\left(\sum_{s \in S} a_{s}^{q^{i}} \frac{\frac{\alpha s}{d q^{n_{s}-i}}}{{ }^{\prime}}\right)=g$.

Notons $E_{1}$ et $E_{2}$ les deux expressions de gauche. Pour voir si un terme de $E_{1}$ se réduit avec un terme de $E_{2}$, on est amené à écrire des égalités du type: $\frac{\alpha_{s}}{d q^{n_{s-i}}}=\beta+\frac{\alpha_{t}}{d q^{n_{t-j}}}$, où $\beta>0,0 \leq i, j \leq n$.

Supposons que: $n_{s}-i=u>0$.

Si $n_{t}-j=-v \leq 0$, alors $\alpha_{s}=q^{u}\left(d \beta+q^{v} \alpha_{t}\right)$ et $q$ divise $\alpha_{s}$ : absurde.

Si $n_{t}-j=v>0$, on distingue les trois cas suivants

a) Si $u=v$, alors $\alpha_{s}-\alpha_{t}=d q^{u} \beta$, donc $\alpha_{s} \neq \alpha_{t}$ et $q$ divise $\alpha_{s}-\alpha_{t}$.

b) Si $u<v$, alors $\alpha_{t}=q^{v-u} \alpha_{s}-d q^{v} \beta$, donc $q$ divise $\alpha_{t}$.

c) Si $v<u$, on trouve que $q$ divise $\alpha_{s}$.

Dans les trois cas, on aboutit aussi à une contradiction.

Ainsi les termes de $E_{1}$ qui se réduisent constituent une série de $\bar{K}\left(\left(\frac{1}{d} G\right)\right)$ 
et les autres se trouvent, par identification dans $g$. D'où: $\sum_{i=0}^{n} b_{i} f^{q^{i}}=h \in \bar{K}\left(\left(\frac{1}{d} G\right)\right)$.

Notation. Dans la suite, $\alpha$ désigne un élément de $G^{-}$non divisible par $q$.

Exemple. Si $\left(a_{i}\right)$ est une suite d'éléments de $\bar{K}$, périodique à partir d'un certain rang, alors la série $f=\sum_{i=0}^{\infty} a_{i} T^{\frac{\alpha}{d q^{i}}}$ de $\tilde{K}$, est algébrique sur $K((G))$.

En effet, quitte à changer un nombre fini de termes, on peut supposer qu'il existe $n \in \mathrm{N}^{*}$, tel que $a_{i+n}=a_{i}$, pour tout $i \in \mathrm{N}$.

On a

$$
\begin{aligned}
f & =\sum_{i=0}^{n-1} \sum_{k=0}^{\infty} a_{i+k n} T^{\frac{\alpha}{d q^{i}+k n}} \\
& =\sum_{i=0}^{n-1} a_{i}\left(\sum_{k=0}^{\infty} T^{\frac{\alpha}{d q^{k n}}}\right)^{q^{-i}} .
\end{aligned}
$$

Soit $g=\sum_{k=0}^{\infty} T^{\frac{\alpha}{d q^{k n}}}$. On a $g^{q^{n}}-g-T^{\frac{q^{n} \alpha}{d}}=0$. On conclut que $f$ est algébrique sur $K((G))$.

2.3. Proposition. La série $f=\sum_{i=0}^{\infty} a_{i} T^{\frac{\alpha}{d q^{i}}}$ de $\tilde{K}$ est algébrique sur $K((G))$ si et seulement si il existe $n \in \mathrm{N}^{*}$ tel que le système $(*) \sum_{t=0}^{n} a_{i+t}^{q^{t}} Y_{t}=0\left(i \in \mathrm{N}^{*}\right)$ admet une solution non triviale dans $K$.

Démonstration. Si $f$ est algébrique, il existe $h \in \bar{K}\left(\left(\frac{1}{d} G\right)\right)$ et des constantes non toutes nulles $c_{0}, \ldots, c_{n} \in K$ tels que: $c_{0} f+c_{1} f^{q}+\ldots+c_{n} f^{q^{n}}=h$.

On trouve: $\sum_{t=0}^{n} \sum_{i=0}^{t} c_{t} a_{i}^{q^{t}} T^{\frac{\alpha q^{-i}}{d}}+\sum_{i=1}^{\infty}\left(\sum_{t=0}^{n} c_{t} a_{i+t}^{q^{t}}\right) T^{\frac{\alpha}{d q^{t}}}=h$.

D'où: $\sum_{t=0}^{n} c_{t} a_{i+t}^{q^{t}}=0$, pour tout $i \in \mathrm{N}^{*}$.

Réciproquement, soit $c_{0}, \ldots, c_{n}$ une solution non triviale du système.

On a: $\sum_{t=0}^{n} c_{t} f^{q^{t}}=\sum_{t=0}^{n} \sum_{i=0}^{t} c_{t} a_{i+t}^{q^{t}} T^{\frac{\alpha q^{t-i}}{d}}$.

2.4. Corollaire. Soit $\left(a_{i}\right)$ une suite d'éléments de $K^{p^{-\infty}}$ et $L=K\left(a_{i}, i \in \mathrm{N}\right)$. 
Si la série $f=\sum_{i=0}^{\infty} a_{i} T^{\frac{\alpha}{d q^{i}}}$ est algébrique sur $K((G))$, alors l'extension $L / K$ est d'exposant fini.

Démonstration. Il existe $n \in \mathrm{N}$ et $c_{0}, \ldots, c_{n-1} \in K$ tels que: $a_{i+n}^{q^{n}}=$ $\sum_{t=0}^{n-1} a_{i+t}^{q^{t}} c_{t}$, pour tout $i \in \mathrm{N}^{*}$. Par récurrence, il existe $s \in \mathrm{N}^{*}$ tel que: $L^{p^{s}} \subseteq K$.

2.5. Corollaire. Soient $f$ et $g$ deux séries de $\tilde{K}$ algébriques sur $K((G))$ telles que: $S(g) \subseteq G^{-}$et $f=\sum_{i=0}^{\infty} a_{i} T^{\frac{\alpha}{d q^{i}}}$. Alors la série: $h=\sum_{i=0}^{\infty} a_{i} g^{\frac{1}{q^{i}}}$ est aussi algébrique sur $K((G))$.

Démonstration. Soit $c_{0}, \ldots, c_{n} \in K$ une solution non triviale de $(*)$ pour $f$. On a: $\sum_{t=0}^{n} c_{t} h^{q^{t}}=\sum_{t=0}^{n} \sum_{i=0}^{t} c_{t} a_{i}^{q^{t}} g^{q^{t-i}}$.

\section{§3. Application aux corps finis}

3.1. Proposition. Soit $f=\sum_{i=0}^{\infty} a_{i} T^{\frac{\alpha}{d q^{i}}} \in \tilde{K}$ une série à coefficients dans un corps fini. Alors $f$ est algébrique sur $K((G))$ si et seulement si la suite $\left(a_{i}\right)$ est périodique.

Démonstration. Soit $c_{0}, \ldots, c_{n} \in K$ une solution non triviale de $(*)$. On peut supposer $c_{n}=1$. Pour $i \in \mathrm{N}$, on pose: $A_{i}=\left(a_{i}, a_{i+1}, \ldots, a_{i+n-1}\right)$. Il existe $i \neq j$ tels que $A_{i}=A_{j}$. D'où: $a_{i+n}=a_{j+n}, \ldots, a_{i+n+k}=a_{j+n+k}$, pour tout $k \in \mathrm{N}$.

\section{EXEMPLES.}

a) La série $f=\sum_{i=0}^{\infty} \frac{\alpha}{T^{q^{i}}}$ est transcendante sur $K((G))$.

b) Définissons les polynômes symétriques:

$$
S_{k}\left(X_{1}, \ldots, X_{n}\right)=\sum_{1 \leq i_{1} \leq \ldots \leq i_{k} \leq n} X_{i_{1}} \ldots X_{i_{k}} .
$$

Soient $a_{1}, \ldots, a_{n}$ des éléments non nuls de $\overline{\mathrm{F}}_{p}$.

La série: $f=\sum_{k=1}^{\infty} S_{k}\left(a_{1}, \ldots, a_{n}\right) T^{\frac{\alpha}{d q^{k}}}$ est algébrique sur $K((G))$.

3.2. Corollaire. Soient $a_{i} \in \mathrm{N}, 0 \leq a_{i} \leq p-1$ pour tout $i \in \mathrm{N}$, et $\bar{a}_{i}$ l'image de $a_{i}$ dans $\mathrm{F}_{p}$, alors la série $f=\sum_{i=0}^{\infty} \bar{a}_{i} T^{\frac{\alpha}{d q^{i}}}$ est algébrique sur $K((G))$ si et seulement si le nombre réel: $\sum_{i=0}^{\infty} \frac{a_{i}}{p^{i}}$ est rationnel. 
3.3. Proposition. Soit $K$ un corps. Les assertions suivantes sont équivalentes.

1) Le corps $K$ est une extension algébrique de son corps premier.

2) Pour tout groupe G, l'ensemble des séries: $f=\sum_{i=0}^{\infty} a_{i} T^{\frac{\alpha}{d q^{i}}}$ de $\tilde{K}$ algébriques sur $K((G))$ coincide avec l'ensemble des séries périodiques.

3) Il existe un groupe $G$ tel que l'ensemble des séries: $f=\sum_{i=0}^{\infty} a_{i} T^{\frac{\alpha}{d q^{i}}} d e \tilde{K}$ algébriques sur $K((G))$ coincide avec l'ensemble des séries périodiques.

Démonstration. 1) $\Longrightarrow 2$ ) Soit $c_{0}, \ldots, c_{n}=1 \in K$ une solution de $(*)$.

On a: $a_{n+1}^{q^{n}}=-\sum_{t=0}^{n-1} a_{1+t}^{q^{t}} c_{t}$. Puisque le corps $F=\mathrm{F}_{p}\left(c_{0}, \ldots, c_{n-1}, a_{1}, \ldots, a_{n}\right)$ est fini, alors $a_{n+1} \in F$.

Par récurrence: $a_{n+i} \in F$, pour tout $i \in \mathrm{N}^{*}$. On conclut par la proposion 3.1 .

2) $\Longrightarrow 3$ ) Evident.

$3) \Longrightarrow 1$ ) Supposons que $K$ ne soit pas algébrique sur $\mathrm{F}_{p}$. Alors $K$ contient un élément $a$ d'ordre multiplicatif infini. La série $f=\sum_{i=0}^{\infty} a^{q^{-i}} T^{\frac{\alpha}{d q^{i}}}$ est algébrique sur $K((G))$, mais $a_{i}=a^{q^{-i}}$ n'est pas périodique.

\section{§4. Exemples}

a) Soient $(v(i), i \in \mathbf{N})$ une suite strictement croissante d'entiers naturels tels que $\lim _{i \rightarrow+\infty}(v(i)-v(i-1))=+\infty$ et $\left(a_{v(i)}, i \in \mathrm{N}\right)$ une suite d'éléments non nuls de $\bar{K}$. La série $f=\sum_{i=0}^{\infty} a_{v(i)} T^{\frac{\alpha}{d q^{(i)}}}$ est transcendante sur $K((G))$.

En effet, supposons que le système $(*)$ admet une solution $c_{0}, \ldots, c_{n}=1 \in K$. Soient $m \in \mathrm{N}$ tel que $n<v(m)-v(m-1)$ et $i=v(m)-n$.

On trouve: $a_{v(m)}^{q^{n}}=0$ : absurde.

b) Soit $\left(a_{i}, i \in \mathrm{N}\right)$ une suite d'éléments de $\bar{K}$ algébriquement indépendants sur $\mathrm{F}_{p}$. Il est facile de voir que pour tout $n \in \mathrm{N}^{*}$, le système: $\sum_{t=0}^{n} a_{i+t}^{q^{t}} Y_{t}=0$, $(i=1+k(n+1), 0 \leq k \leq n)$ est de Cramer.

Donc la série: $f=\sum_{i=0}^{\infty} a_{i} T^{\frac{\alpha}{d q^{i}}}$ est transcendante sur $K((G))$. 
c) Soit $0 \neq x \in \bar{K}$. La série: $f=\sum_{i=0}^{\infty} x^{i} T^{\frac{\alpha}{d q^{i}}}$ est algébrique sur $K((G))$ si et seulement si $x$ est algébrique sur $\mathrm{F}_{p}$.

" $\Rightarrow$ " Soit $n$ un entier pour lequel le système $(*)$ admet une solution non triviale dans $K$. Le système: $\sum_{t=0}^{n} x^{(i+t) q^{t}} Y_{t}=0,(1 \leq i \leq n+1)$ a pour déterminant: $P(x)=x^{1+2 q+3 q^{2}+\ldots+(n+1) q^{n}} V\left(x, x^{q}, \ldots, x^{q^{n}}\right)$, où $V$ désigne le déterminant de Vandermonde. Doù: $P(x)=0$.

" $\Leftarrow$ " Il existe $n \in \mathrm{N}^{*}$ tel que: $x^{n}=1$. Le résultat découle de la proposition 3.1.

d) Soit $\left(u_{i}, i \in \mathrm{N}\right)$ une suite récurrente linéaire d'éléments de $\overline{\mathrm{F}}_{p}$. D'après [3] th.8, il existe un entier $s \geq 1$, tel que pour tout $r \in\{0,1, \ldots, s-1\}$, il existe des éléments $a_{i}, b_{i} \in \overline{\mathrm{F}}_{p}, 1 \leq i \leq t$, dépendants de $r$, vérifiant: $\forall k \in \mathrm{N}$, $u_{k s+r}=\sum_{i=1}^{t} a_{i} b_{i}^{k}$.

Soit $f=\sum_{i=0}^{\infty} u_{i} T^{\frac{\alpha}{d q^{r}}}$. On a: $f=g_{0}+\ldots+g_{s-1}$, où $g_{r}=\sum_{k=0}^{\infty} u_{k s+r} T^{\frac{\alpha}{d q^{k s+r}}}=$ $\sum_{i=1}^{t} a_{i}\left(\sum_{k=0}^{\infty}\left(b_{i}^{q^{r}}\right)^{k} \frac{\alpha}{d u^{k}}\right)^{q^{-r}}$, avec $u=q^{s}$. D'après l'exemple (c), la série $g_{r}$ est algébrique sur $K((G))$. Il en est de même pour $f$.

\section{§5. Polynômes minimaux}

On suppose que $K$ est algébriquement clos. Soit $f$ une série de $\tilde{K}$ algébrique sur $K((G))$ et vérifiant les hypothèses du lemme 2.2. Il existe $n \in \mathrm{N}^{*}, h \in \bar{K}\left(\left(\frac{1}{d} G\right)\right)$ et des constantes $c_{0}, \ldots, c_{n-1} \in K$ tels que $f$ soit racine du polynôme:

$$
P(X)=X^{q^{n}}+c_{n-1} X^{q^{n-1}}+\ldots+c_{1} X^{q}+c_{0} X+h .
$$

On suppose que $c_{0} \neq 0$. Les racines de $P(X)$ sont alors distinctes.

5.1. Proposition. L'extension $K((G))(f) / K((G))$ est abélienne de degré $p^{s}$, où $s \in \mathrm{N}^{*}$ et son groupe de Galois est une somme directe de s sous groupes cycliques d'ordre $p$.

Démonstration. Les racines de $P(X)$ sont les $f+x, x$ est racine du polynôme: $X^{q^{n}}+\ldots+c_{0} X$ dans $K$. Il en résulte que tous les facteurs irréductibles de $P(X)$ ont le même degré et l'extension $K((G))(f) / K((G))$ est galoisienne de degré $p^{s}$. Un élément de son groupe de Galois est de la forme $\tau_{x}$, avec $\tau_{x}(f)=f+x$. 
5.2. Lemme. Soient $K$ un corps de caractéristique $p \neq 0$ et $\left(x_{i}, i \in \mathrm{N}^{*}\right)$ une suite d'éléments de $K$. On définit la suite de polynômes $P_{i}(X)$ par:

$$
P_{1}(X)=X^{p}-x_{1}^{p-1} X \text {, et pour } i \geq 2, P_{i}(X)=P_{i-1}^{p}(X)-P_{i-1}^{p-1}\left(x_{i}\right) P_{i-1}(X) \text {. }
$$

Alors pour: $0 \leq \alpha_{1}, \alpha_{2}, \ldots, \alpha_{i} \leq p-1 ; \alpha_{1} x_{1}+\ldots+\alpha_{i} x_{i}$ est racine de $P_{i}(X)$.

Démonstration. Par récurrence, en remarquant que $P_{i}$ est additif.

Reprenons les notations introduites au début de ce $\S$. Soit $S=$ $\operatorname{Gal}(K((G))(f) / K((G)))$. Alors $S=\bigoplus_{i=1}^{s} C_{i}$, où $C_{i}=<\tau_{i}>$ est un groupe cyclique d'ordre $p$. On a: $\tau_{i}(f)=f+x_{i}$, où $x_{i} \in K$. Les conjugués de $f$ sont les $f+\alpha_{1} x_{1}+\ldots+\alpha_{s} x_{s}$, avec $0 \leq \alpha_{i} \leq p-1$. Puisque l'égalité $\tau_{1}^{\alpha_{1}} \ldots \tau_{s}^{\alpha_{s}}=i d$, pour $0 \leq \alpha_{i} \leq p-1$, implique $\alpha_{1}=\ldots=\alpha_{s}=0$, alors $x_{1}, \ldots, x_{s}$ sont linéairement indépendants sur $\mathrm{F}_{p}$. Donc les $f+\alpha_{1} x_{1}+\ldots+\alpha_{s} x_{s}$ sont des racines distinctes du polynôme: $P_{s}(X-f)=P_{s}(X)-P_{s}(f)$. D'où:

5.3. Proposition. Le polynôme minimal de $f$ sur $K((G))$ est égal à:

$$
P_{s}(X)-P_{s}(f)
$$

EXEMPLE. On suppose $p=2$. Soit $f=\sum_{i=0}^{\infty} a_{i} T_{2^{i}}$, avec $a_{0}=a_{1}=1, a_{2}=0$ et $a_{i+3}=a_{i}$. On a: $a_{i}+a_{i+1}+a_{i+2}=0$.

Le polynôme minimal de $f$ est $X^{4}+X^{2}+X+T^{4 \alpha}$.

\section{RÉFÉRENCES}

1. N.L. Alling, Foundation of analysis over surreal number fields, North-Holland Math. Stud. $141,1987$.

2. J.P. Allouche, Automates finis en théorie de nombres, Exposition. Math. 5 (1987), 239-266.

3. J.P. Bezivin, Suites récurrentes linéaires en caractéristique non nulle, Bull. Soc. Math. France 115 (1987), 227-239.

4. F.J. Rayner, An algebraically closed field, Glasgow Math. J. 9 (1968), 146-151.

5. F.J. Rayner, Algebraically closed fields analogous to fields of Puiseux theorem, J. London Math. Soc. (2) 8 (1974), 504-506.

6. P. Ribenboim, Fields algebraically closed and others, Manuscripta Math. 75 (1992), 115-150.

7. D. Stephanescu, A method to obtain algebraic elements over $K((T))$ in positive characteristic, Bull. Math. Soc. Sci. Math. R. S. Roumanie 26 (74) (1982), 78-91.

8. D. Stephanescu, On meromorphic formal power series, Bull. Math. Soc. Sci. Math. R. S. Roumanie 27 (75) (1983), 170-178.

DÉPARTEMENT DE MATHÉMATIQUES FACULTÉ DES SCIENCES 5000 MONASTIR

TUNISIE 